\title{
Knockdown of hsa_circ_0000729 Inhibits the Tumorigenesis of Non-Small Cell Lung Cancer Through Mediation of miR-I28I/FOXO3 Axis
}

\section{Xiao Xie \\ Fangbao Ding \\ Haibo Xiao}

Department of Cardiothoracic Surgery, Xinhua Hospital Affiliated to Shanghai Jiao Tong University School of Medicine, Shanghai, 200092, People's Republic of China
Correspondence: Fangbao Ding; Haibo Xiao

Department of Cardiothoracic Surgery, Xinhua Hospital Affiliated to Shanghai Jiao Tong University School of Medicine, No. 1665 Kongiiang Road, Shanghai, 200092, People's Republic of China Email dingfangbao@xinhuamed.com.cn; xiaohaibo@xinhuamed.com.cn
Background: Non-small cell lung cancer (NSC C) is a subty ${ }_{\mathrm{P}}$ of ang cancer which seriously threatens the health of people. Circula VNAs ircRNAs) are endogenous RNAs which have stable closed structure; they kno o be in $\mathrm{c}$ ed in tumorigenesis of NSCLC. Meanwhile, hsa_circ_00007 was repor to e upregulated in NSCLC. Nevertheless, the function of hsa_ci 000 in NSCL 9 remains unclear.

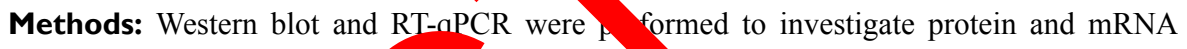
levels, respectively. CCK- 8 ay was performed cest the cell viability and cell death was investigated by flow cytor try. NSCLC ell pyroptosis was observed by electron microscope. In addition, the mi ation and in sion of NSCLC cells were detected by wound healing and transwell assay. relatio among hsa_circ_0000729, miR-1281 and FOXO3 was explored by than iferase roponer assay and RNA pull-down.

Results: Hsa_cir $0000^{\circ}$ found to be upregulated in NSCLC cells, and hsa circ_00 a knoc abviously suppressed the proliferation of NSCLC cells through indy ng py tosis. addition, silencing of hsa_circ_0000729 notably inhibited the invaand $\mathrm{w}$ mion of SCLC cells. Meanwhile, hsa_circ_0000729 could bind with miR128 FOXO3 was directly targeted by miR-1281. Moreover, the anti-tumor effect of hsa_cir 900729 siRNAs on NSCLC was markedly reversed by miR-1281 antagomir. Furthermo silencing of hsa_circ_0000729 inhibited the tumor growth of NSCLC in vivo. onclusion: Knockdown of hsa_circ_0000729 inhibits the tumorigenesis of NSCLC thr hediation of miR-1281/FOXO3 axis. Thus, hsa_circ_0000729 might be served as a crucial mediator in NSCLC.

Keywords: hsa_circ_0000729, miR-1281, FOXO3, pyroptosis

\section{Introduction}

Non-small cell lung cancer (NSCLC) is a type of lung malignant tumor. In addition, NSCLC is reported to be the leading cause of tumor-related mortality in the world. ${ }^{1}$ Nowadays, the major treatments for NSCLC are radiotherapy, surgery and chemotherapy, while the prognosis of patients with NSCLC remains not ideal. ${ }^{2,3}$ Although great studies have been made to treat NSCLC over 30 years, the survival rate of patients is still at low level due to the recurrence and metastasis of tumor. ${ }^{4}$ Thereby, it is essential to explore new strategies against NSCLC.

Circular RNAs (CircRNAs) are endogenous RNAs which have stable closed structure. ${ }^{5}$ It has been revealed that circRNAs participate in the cancer progression. For instance, Yi et al found that hsa_circ_0001806 overexpression could increase 
the growth, migration and invasion of NSCLC cells via mediation of miR-1182/NOVA2 axis; ${ }^{6}$ Wang et al indicated that circ-PTEN upregulation could suppress the proliferation of NSCLC cells through activation of PTEN. ${ }^{7}$ Meanwhile, it has been revealed that hsa_circ_0000729 was significantly upregulated in lung adenocarcinoma (LUAD). ${ }^{8}$ LUAD is the main subtype of NSCLC. ${ }^{9}$ Nevertheless, the function of hsa_circ_0000729 in NSCLC remains largely unknown.

Recent studies indicated that pyroptosis plays a crucial role in cellular process. ${ }^{10,11}$ Meanwhile, pyroptosis is a programmed cell death which is majorly regulated by caspase $1 .^{12,13}$ In addition, caspase 1 is often upregulated when the growth of NSCLC cells is inhibited, suggesting that the promotion of pyroptosis might be associated with the progression of NSCLC. ${ }^{14}$ Therefore, it is necessary to investigate the correlation between hsa_circ_0000729 and pyroptosis in NSCLC cells with the purpose of exploring new effective strategies against NSCLC.

This study sought to investigate the function of hsa_circ_0000729 in NSCLC. We hope this research would shed new lights on exploring the new methods for the treatment of NSCLC.

\section{Materials and Methods}

\section{Cell Culture}

NSCLC cell lines (A549, NCI-H1650 and 1-H129) and human normal lung epithelial BEAS-2P celı ve cre pur chased from Chinese Academy $O$ sciences Thanghai, China). In addition, cells were ant ned in RPh 1640 medium (Thermo Fisher Scintific, Wa m, MA, USA) containing FBS $(10 \%$, Tb mo Fisher Scien. $)$ ) and penicillin $(100 \mathrm{U} / \mathrm{mL})$ in a ncubat at $37^{\circ} \mathrm{C}$.

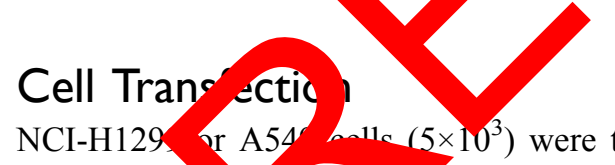

NCI-H129 or A54 $11_{c}\left(5 \times 10^{3}\right)$ were transfected with hsa_circ_0000 siRNA1 (s1-hsa_circ_0000729-1), hsa_circ_0000729 St_ JA2 (si-hsa_circ_0000729-2), hsa_circ_0000729 siR_A3 (si-hsa_circ_0000729-3) or negative control (NC; siRNA-ctrl) for $24 \mathrm{~h}$ by using Lipofectamine $^{\circledR} 2000$ (Invitrogen). Hsa_circ_0000729 siRNA1, siRNA2, siRNA3 and siRNA-ctrl were purchased from Genepharma (Shanghai, China). After 24 $\mathrm{h}$ of transfection, cells were harvested of use in the subsequent analysis.

For miR-1281 transfection, NSCLC cells were transfected with miR-1281 agomir, miR-1281 antagomir or NC by using Lipofectamine ${ }^{\circledR} 2000$ (Thermo Fisher Scientific). MiR-1281 agomir, antagomir and NC were obtained from Genepharma. After $24 \mathrm{~h}$ of transfection, cells were harvested for use in the subsequent analysis.

For hsa_circ_0000729 overexpression, NCI-H1650 cells were transfected with pcDNA3.1 or pcDNA3.1-hsa_circ_0000729 for $24 \mathrm{~h}$ by using Lipofectamine $^{\circledR} \quad 2000 \quad$ (Thermo Fisher Scientific). pcDNA3.1 and pcDNA3.1-hsa_circ_0000729 were obtained from Genepharma.

\section{CCK-8 Assay}

NSCLC cell viability in each gro was evalu ted using a CCK-8 assay kit (Beyot e, Shang i Chir). In brief, NCI-H1299, A549 or N $1-H 165^{\circ}$ cells (. o were treated with NC or siha c of $0729-3$. After 24,48 or 72 $\mathrm{h}$ of incubation ells wer reated 1 th CCK-8 reagents $(10 \mu \mathrm{L})$ for 1 . absorban $450 \mathrm{~nm})$ was measured by a microplate reac

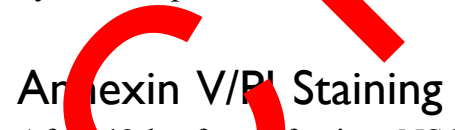

Aft $48 \mathrm{~h}$ of tra fection, NSCLC cells were trypsinized, washe ith $\mathrm{n}^{1}$ sphate buffered saline and resuspended in ain V Binding Buffer. Then, cells were stained with 5 Anmuin $\mathrm{V}$ and $5 \mu \mathrm{L}$ propidium (PI) in the dark for 15 min. Cells were analyzed by flow cytometer (BD iosciences, Franklin Lake, NJ, USA). In addition, the data were analyzed using FACS (BD Biosciences) with FlowJo (v10.6.2; BD Biosciences).

\section{Reverse Transcription-Quantitative PCR (RT-qPCR)}

Total RNA was extracted from liver cancer cell lines using TRIzol reagent (TaKaRa, Tokyo, Japan) according to the manufacturer's protocol. First-strand cDNA was synthesized using the PrimeScript RT reagent Kit (Takara). After that, RT-qPCR was performed by using the SYBR premix Ex Taq II kit (ELK bioscience, Wuhan, China). Real-Time qPCRs were performed in triplicate. The protocol of amplification was as follows: 2 minutes at $94^{\circ} \mathrm{C}$, followed by 35 cycles $\left(30 \mathrm{~s}\right.$ at $94^{\circ} \mathrm{C}$ and $45 \mathrm{~s}$ at $\left.55^{\circ} \mathrm{C}\right)$. The following primer pairs were used for RT-qPCR: hsa-circ -0000729 forward, 5'-AAGTGAGCATGTGTGTGCAGG $-3^{\prime}$ and reverse, 5'-TCCACCTGCTCACTTGCATG-3'; miR-1281 forward, 5'-TCGGTCGCCTCCTCCTC-3' and reverse, 5'-CTCAACTGGTGTCGTGGAGTC-3'; $\beta$-actin forward, 5'-GTCCACCGCAAATGCTTCTA-3' and 
reverse, 5'-TGCTGTCACCTTCACCGTTC-3'; U6 forward, 5'-CTCGCTTCGGCAGCACAT-3' and reverse, 5'AACGCTTCACGAATTTGCGT-3'. The levels of gene were quantified using the $2^{-\Delta \Delta t}$ method. $\beta$-actin or U6 was used an internal reference.

\section{Western Blot}

Total protein was isolated from cell lysates or tissues by using RIPA buffer. The concentration of protein was detected with a BCA protein kit (Thermo Fisher Scientific). Then, proteins (40 $\mu \mathrm{g}$ per lane) were separated with 10\% SDS-PAGE gel and then transferred into polyvinylidene fluoride (PVDF, Thermo Fisher Scientific) membranes. The PVDF membranes were incubated overnight with primary antibodies after being blocked with $3 \%$ skim milk for $1 \mathrm{~h}$. The primary antibodies were as follows: anti-FOXO3 (1:1000), anti-cleaved caspase 1 (1:1000), anti-GSDME-N (1:1000) and anti- $\beta$-actin $(1: 1000)$. After that, the membrane was incubated with goat anti-rabbit secondary antibody (HRP-labeled, 1:5000) for $1 \mathrm{~h}$. All antibodies were obtained from Abcam (Cambridge, MA, USA). ECL reagent (Thermo Fisher Scientific) was used to visualize the protein bands. ImageJ software (version 2.0) was performed to quantify the intensity of the

\section{Transwell Assay}

The upper chamber was pre-treated w (This procedure was not included NSCLC cells $\left(4 \times 10^{4}\right)$ were sus ided $\mu \mathrm{L}$, Thermo Fisher Scientifi vithout FB The lower chamber was supplemented with a culture modium containing 20\% FBS. Fo swing incub on for $24 \mathrm{~h}$, the migrated or invade cells yere fixed and stained with crystal violet $(0.2 \mathrm{a}$ ) for $\mathrm{min}$. The result was observed by a microse (mas acation 200$)$.

\section{Wou d He ina Assay}

A549 cell e plated at a density of $2 \times 10^{5}$ cells per well. When A549 1/s reached $80 \%$ confluence, a wound area was scratched in the cell monolayer using a pipette tip. After that, cells were treated with sihsa_circ_0000729-3 or sihsa_circ_0000729-3 + miR-1281 antagomir for $24 \mathrm{~h}$ at $37^{\circ} \mathrm{C}$. Images were captured at 0 and $24 \mathrm{~h}$ under a fluorescence microscope (Leica Microsystems, Inc.).

\section{ELISA}

The levels of IL-18 and IL-1 $\beta$ in supernatants of NSCLC cells were investigated by ELISA detection kit
(Multisciences (Lianke) Biotech Co., Ltd, Hangzhou, China) according to the manufacturer's instructions.

\section{Dual-Luciferase Reporter Assay}

The partial sequences of hsa_circ_0000729 and the FOXO3 3'-UTR containing the sites of miR-1281/miR$1178 / \mathrm{miR}-324-5 \mathrm{p} / \mathrm{miR}-767-3 \mathrm{p}$ were synthesized by GenePharma. The aforementioned sequences were cloned into the pmirGLO vectors (Promega, Madison, WI, USA) for establishment of wild-type (WT) or mutant (MUT) reporter hsa_circ_0000729 and EDuaz vectors. The WT or MUT hsa_circ_0000729 yor was ransfected into cells along with miR-128 iR-1178/mi -324-5p/miR767-3p agomir using pofecta ine 200 reagent. The data were quantifier oy normalizin cenilla luciferase activity.

\section{Scannip}

NSCLC cells ere fixed and rinsed three times. $\mathrm{Su}^{1}$ ently, cells vere dehydrated through ethanol and ried by using the tertiary butanol method. After that, amples wer mounted and dried in a silica gel vacuum iccator. nally, the morphology was observed by a sca. olectron microscope (SEM, $20 \mathrm{kV})$.

\section{RNÁ Pull-Down Assay}

Probe-control or probe-hsa_circ_0000729 was transcribed and labeled with a Biotin RNA Labeling Mix (Roche, Basel, Switzerland). Cells were lysed with Poly-lysis buffer (ELK bioscience), washed with PBS and centrifuged at $1000 \times \mathrm{g}$ for $5 \mathrm{~min}$ at $4^{\circ} \mathrm{C}$. Secondary structure formation in the biotin-labeled RNAs was induced with RNA structure buffer (Thermo Fisher Scientific). Streptavidin beads (75 $\mu \mathrm{L}$; Thermo Fisher Scientific) were washed and incubated overnight. After that, streptavidin bead-RNA complexes were obtained by separating the mixture. Then, cell lysates $\left(5 \times 10^{7}\right.$ cells $)$ were added to the complexes and incubated for $1 \mathrm{~h}$. Following incubation, the mixture was separated again, and the supernatant of cell lysates was utilized to detect the enrichment of miR-1281. Meanwhile, RT-qPCR was performed to investigate the enrichment of miR-1281.

\section{In vivo Study}

BALB/c nude mice ( $n=8 ; 6-8$ weeks old) were obtained from Vital River (Beijing, China). The protocols for animal care and use of laboratory animals were in accordance with ethical committee of Xinhua Hospital Affiliated to Shanghai Jiao 
Tong University School of Medicine (No. XH20200725). A549 cells transfected with $50 \mathrm{nM}$ si-hsa_circ_0000729-3 were subcutaneously transplanted in mice. The tumor volume was investigated once a week as the following formula: length $\times$ width $\times$ width. At end of the study, mice were sacrificed for tumor tissue collection. All in vivo experiments were performed according to the guideline of National Institutes of Health for the care and use of laboratory animals.

\section{Statistical Analysis}

Data were presented as the mean \pm standard deviation. CCK8 assay was performed in quintuplicate. Flow cytometry, Western blot, RT-qPCR, luciferase activity test and ELISA were repeated in triplicate. The other experiments were performed three times. In addition, Student's $t$-test was used to compare the difference between two groups. Oneway analysis of variance and Tukey's post hoc tests were used for comparisons between $\geq 3$ groups. $\mathrm{P}<0.05$ was considered to indicate a statistically significant difference.

\section{Results}

Knockdown of hsa_circ_0000729 Inhibited the Proliferation of NSCLC

\section{Cells}

The background information of hsa_circ_0000729 is presented in Figure 1A. To investigate the expression of hsa circ_0000729 in NSCLC cell lines, RT-qPCR was performed. As indicated in Figure 1B, the level of hsa_circ_0000729 in NSCLC cells (A549, NCI-H1650 and NCI-H1299) was much higher than that in BEAS-2B cells. Sino circ_0000729 expression was much higher in A5 and NC. 1299 cells, these two cell lines were selecte f use in th following experiments. Additionally, th Mevel os sa circ 000729 in NSCLC cells was marke decre ed by circ_0000729 siRNA1, siRNA2 or siR 3 (P sure 1C and D). Meanwhile, A549 and NCI 299 w more asceptible to si-hsa _circ_000072 $\sigma$, mpared wit _er two siRNAs. Thus, sihsa_circ_0000729-3 selected of use in the subsequent

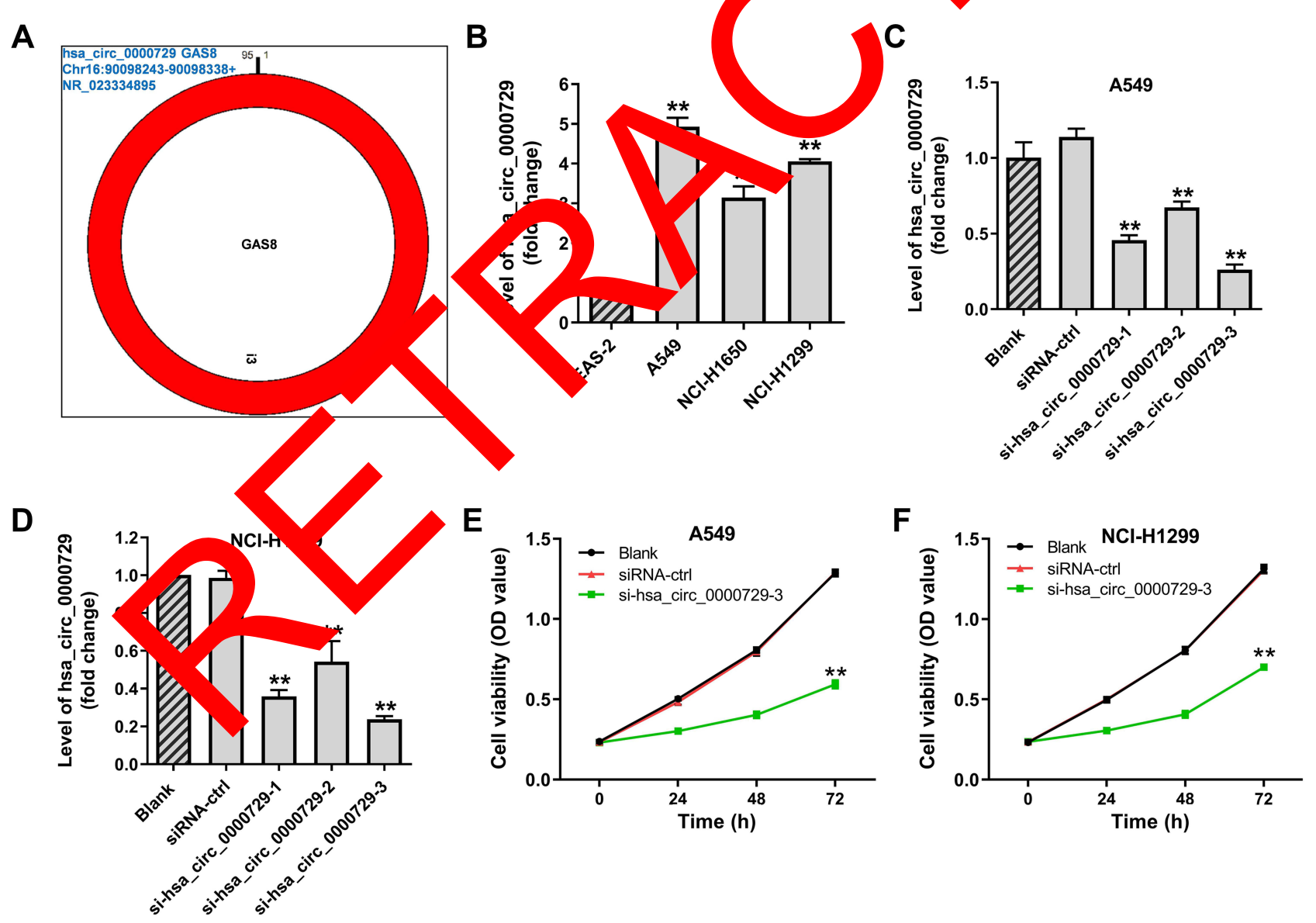

Figure I Knockdown of hsa_circ_0000729 inhibited the proliferation of NSCLC cells. (A) The background information of hsa_circ_0000729 was presented. (B) The expression of hsa_circ_0000729 in BEAS-2B, A549, NCl-HI650 or NCl-HI299 cells was detected by RT-qPCR. (C and D) A549 or NCl-HI299 cells were transfected with siRNA-ctrl (NC), hsa_circ_0000729 siRNAI, siRNA2 or siRNA3. Then, the expression of hsa_circ_0000729 in NSCLC cells was investigated by RT-qPCR. (E and F) NClHI299 cells were transfected with NC or si-hsa_circ_0000729-3. The viability of NSCLC cells was tested by CCK-8 assay. **P < 0.0 I compared with control. 
experiments. CCK8 data suggested si-hsa_circ_0000729-3 notably inhibited the viability of NSCLC cells (Figure 1E and F). Taken together, knockdown of hsa_circ_0000729 inhibited the proliferation of NSCLC cells.

\section{Hsa_circ_0000729 siRNA Greatly Induced Pyroptosis in NSCLC Cells}

In order to explore the manner of death in NSCLC cells, flow cytometry was used. As shown in Figure 2A-D, the percentage of cell death was significantly upregulated by hsa circ_0000729 knockdown, and the ratio of PI positive cells was also notably increased by hsa_circ_0000729 siRNA3. The data suggested hsa_circ_0000729 knockdown might induce pyroptosis in NSCLC cells. Next, SEM was used to investigate if si-hsa_circ_0000729-3 could induce pyroptosis in NSCLC cells. The data revealed the membrane breakage and pyroptosis body in A549 or NCI-H1299 cells were significantly increased by si-hsa_circ_0000729-3 (Figure 2E and F). These phenomena were the typical feature of pyroptosis. ${ }^{15}$ Thus, these data suggested that si-hsa_circ_0000729-3 greatly induced pyroptosis in NSCLC cells. Since A549 cells were more sensitive to si-hsa_circ_0000729-3 treatment (According to the result of CCK8, the inhibition rate of A549 cells after transfeo f hsa_circ_0000729 siRNA3 was 54\%, while the inhibitio rate of NCI-H1299 cells was 46\%), A549 cells were rected of in the following experiments.

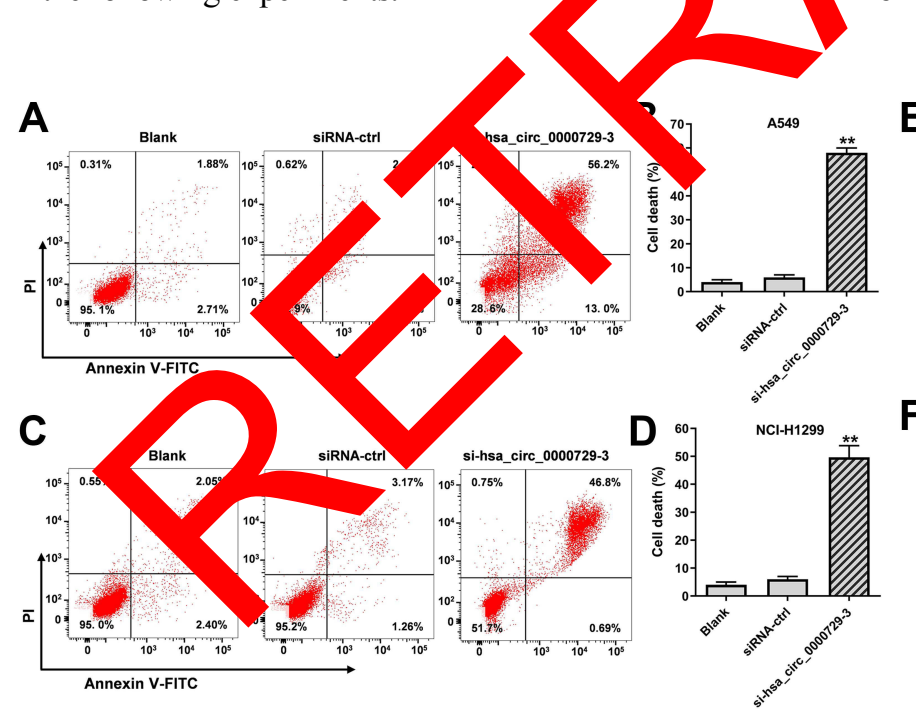

\section{Hsa_circ_0000729 Could Bind with miR- $|28|$}

In order to explore the mechanism underlying the function of hsa_circ_0000729 in NSCLC tumorigenesis, the database (https://circinteractome.nia.nih.gov/) was used. The data revealed that hsa_circ_0000729 had a putative binding site with miR-1281 (Figure 3A). Also, miR-1178, miR-324-5p and miR-767-3p might be the downstream miRNAs of hsa_circ_0000729. In addition, the relative luciferase activity in WT-hsa_circ_0000729 was obviously inhibited by miR-1281 agomir, while miR-1281 agomir a non ect the luciferase activity in MUT-hsa_circ_00e 29 (Figure - ). Meanwhile, the luciferase activity in WT/\ T-hsa_cir 0000729 was rarely affected by doy egulation or $\mathrm{iR}$ 18, miR-324-5p or miR-767-3p ( $\mathrm{Su}_{\mathrm{L}}$ ement Figure $\mathrm{A}-\mathrm{C}$ ). Next, the level of miR-1281 iv $\mathrm{SCL}$ is was sificantly upregulated by miR-1281 mir but, hibi by miR-1281 antagomir (Figure C C). In dition, the result of pulldown indicated the enri-nt of mik 281 was notably higher in probe-hsa circ_0000729, compared with that in control (Figure 3D). zased on th above data, hsa_circ_0000729 possibly bind miR-12

we explore the potential target of miR-1281 Targetscan (http://www.targetscan.org/vert 72/) online tool and the database suggested miR-1281 had binding sites with FOXO3 (Figure 3E). Additionally, the
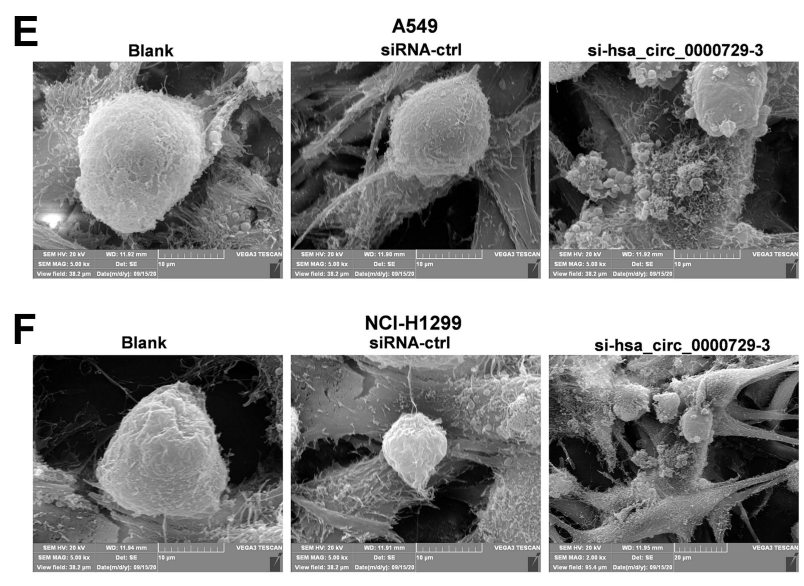

Figure 2 Si-hsa_circ_0000729-3 greatly induced pyroptosis in NSCLC cells. (A and B) The percentage of A549 cell death was tested by flow cytometry. (C and D) The percentage of $\mathrm{NCl}-\mathrm{H} 1299$ cell death was tested by flow cytometry. (E and $\mathbf{F}$ ) The pyroptosis of NSCLC cells was observed by SEM. **P < $0.0 \mathrm{I}$ compared with control. 


\begin{tabular}{|c|c|c|c|}
\hline $\begin{array}{l}\text { CircRNA } \\
\text { miRNA ID }\end{array}$ & $\begin{array}{l}\text { CircRNA-miRNA } \\
\text { pairing }\end{array}$ & $\begin{array}{c}\text { CircRNA } \\
\text { start }\end{array}$ & $\begin{array}{c}\text { CircRNA } \\
\text { end }\end{array}$ \\
\hline 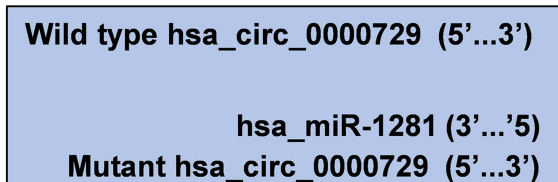 & 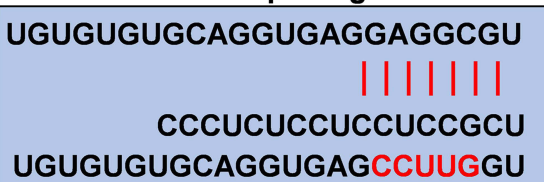 & 74 & 80 \\
\hline
\end{tabular}

B

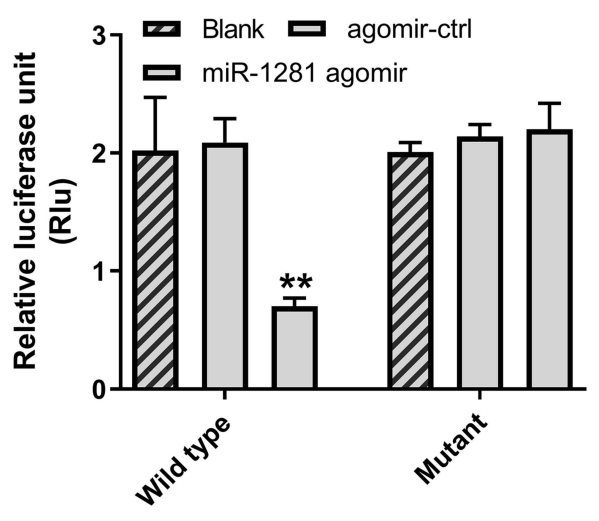

C

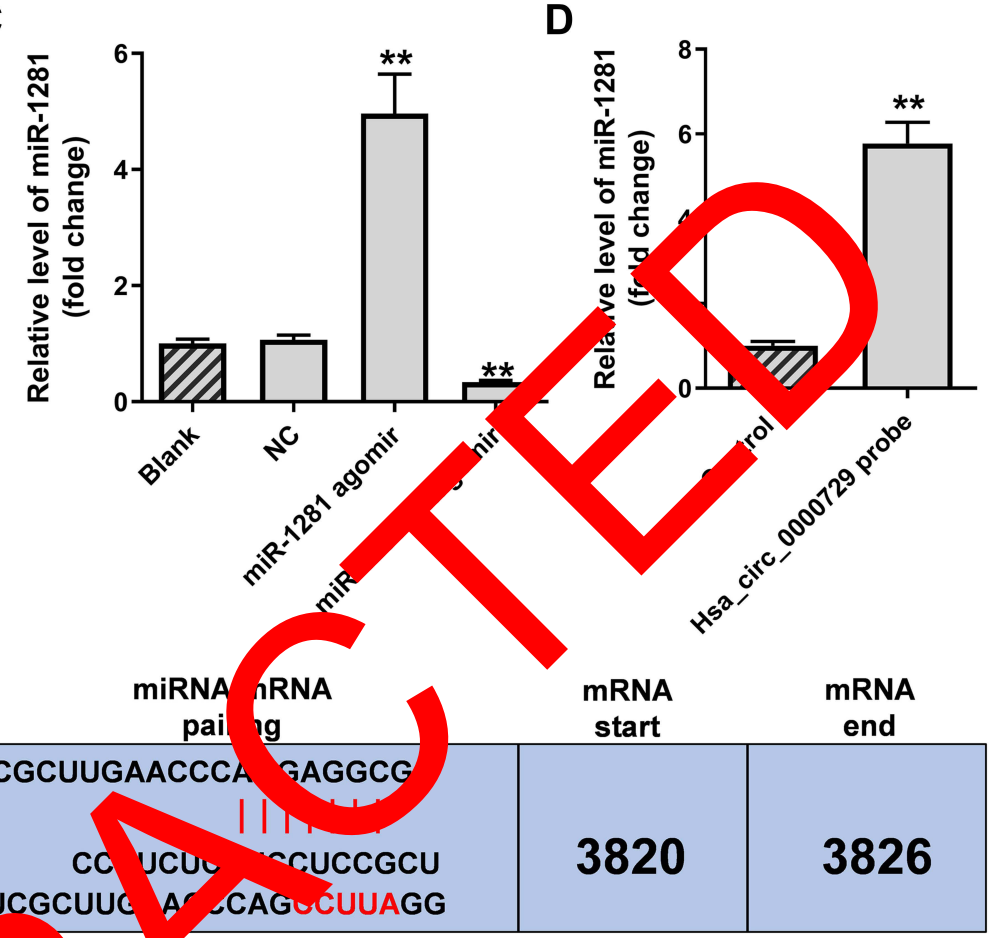

\section{$\mathbf{F}$}
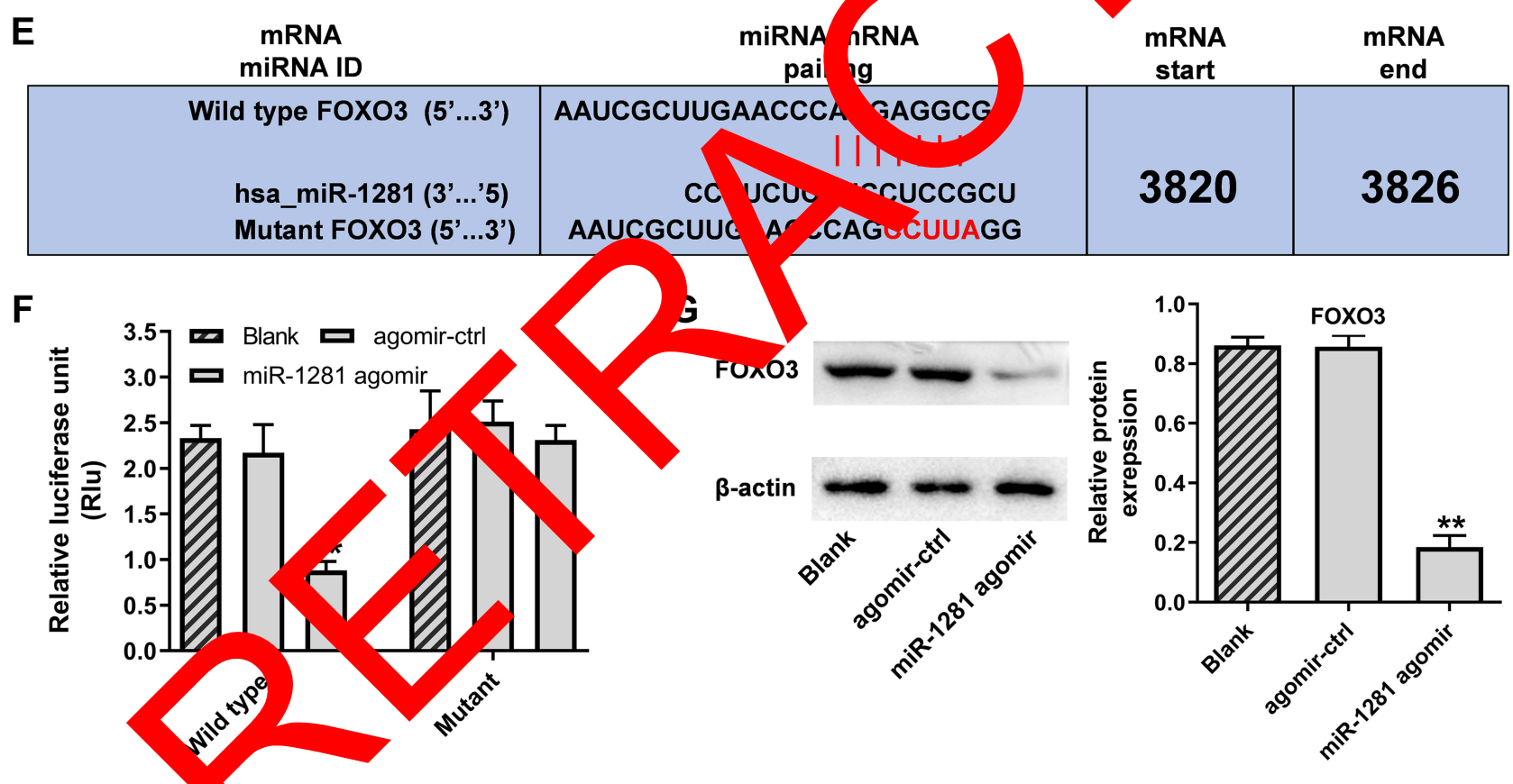

Figure 3 Hsa_circ_0000, could bind with miR-128I. (A) Circular RNA Interactome was used to predict the downstream miRNA of hsa_circ_0000729. (B) The relative luciferase activity in WT/MO sa_circ_0000729 was tested by dual luciferase reporter assay. (C) NSCLC cells were transfected with NC, miR-I28I agomir or miR-I28I antagomir. Then, the efficiency of cell transfection was detected by RT-qPCR. (D) The enrichment of miR-I28I was investigated by RNA pull-down. (E) FOXO3 was identified to be the direct target of miR-I 281 by using targetscan online tool. (F) The relative luciferase activity in WT/MUT-FOXO3 was tested by dual luciferase reporter assay. (G) The protein level of FOXO3 in NSCLC cells was detected by Western blot. The relative expression was quantified by normalizing to $\beta$-actin. **P < 0.01 compared with control.

relative luciferase activity in WT-FOXO3 was significantly reduced in the presence of miR-1281 agomir (Figure 3F), and the protein level of FOXO3 in NSCLC cells was markedly decreased by miR-1281 agomir (Figure 3G). In summary, miR-1281 could bind with FOXO3. 


\section{Hsa_circ_0000729 Knockdown-Induced Pyroptosis in NSCLC Cells Was \\ Significantly Reversed by miR-I28I}

\section{Antagomir}

To explore the mechanism by which hsa_circ_0000729 induced pyroptosis in NSCLC cells, Western blot was used. As demonstrated in Figure 4A-D, hsa_circ_0000729 siRNA significantly decreased the protein level of FOXO3 and upregulated the levels of cleaved caspase 1 and GSDME-N in NSCLC cells, while these phenomena were partially reversed by miR-1281 antagomir. In addition, hsa_circ_0000729 silencing-induced upregulation of IL-1 $\beta$ and IL-18 was notably abolished by miR-1281 antagomir (Figure 4E and F). Furthermore, miR-1281 antagomir partially reversed hsa_circ_0000729 knockdown-induced cell growth inhibition of NSCLC (Figure 4G-I). Since cleaved caspase 1, GSDME-N and IL-18 were known to be key mediators in pyroptosis, ${ }^{16,17}$ it could be suggested that hsa_circ_0000729 knockdown-induced pyroptosis in NSCLC cells was through sponging miR-1281.

\section{Silencing of Hsa_circ_0000729 Inhibited the Migration and Invasion of NSCLC Cells Through Sponging miR-I28I}

In order to further confirm the relation between hsa circ_0000729 and miR-1281 in NSCLC, transwell and wound healing assay were performed. As shown in Figure $5 \mathrm{~A}-\mathrm{C}$, the migration and invasion of NSCLC cells were notably inhibited by si-hsa_circ_0000729-3, while this phenomenon was partially reversed by miR1281 antagomir. Therefore, these results indicated that silencing of hsa_circ_0000720 mintur the migration and invasion of NSCLC cell arough spon ng miR-1281.

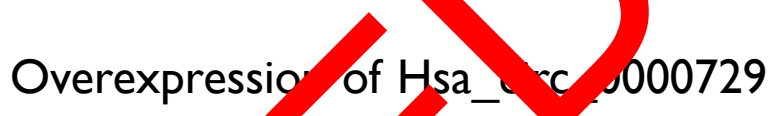

Increased the jiab icy, Migration and Invasion $\mathrm{NCl} \cdot 1650$ Cells

To furth ca frm the fu. on of hsa_circ_0000729 in NSCLC in vitro, CCI-H1650 cells were transfected with NA.1-hsa_circ_000729. As revealed in upplementary Figure 2A, the level of hsa_circ_0000729

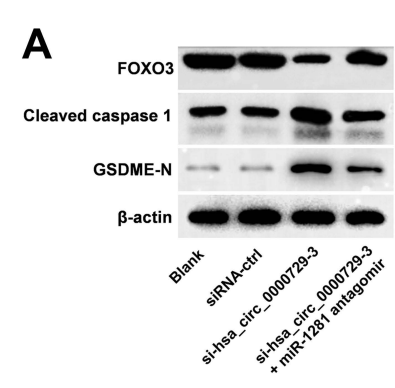

$\mathbf{F}$

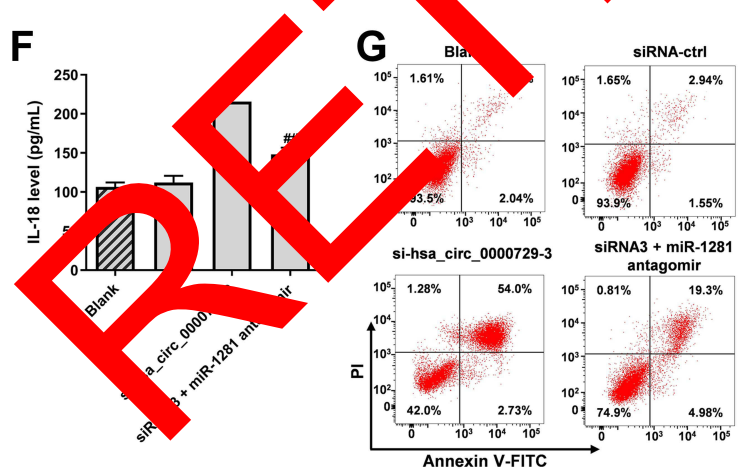

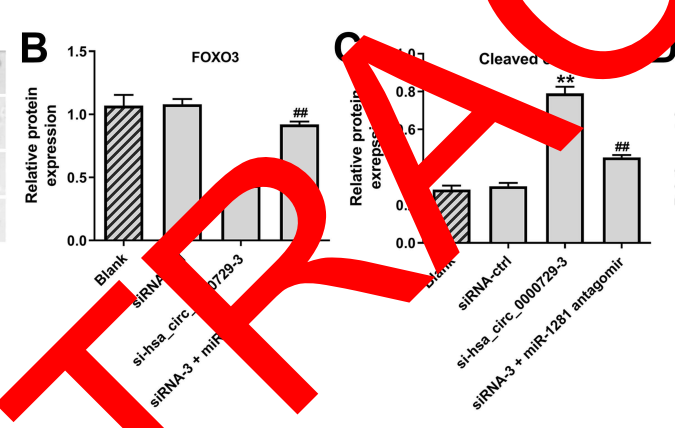

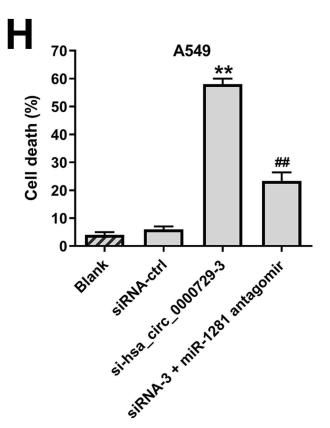

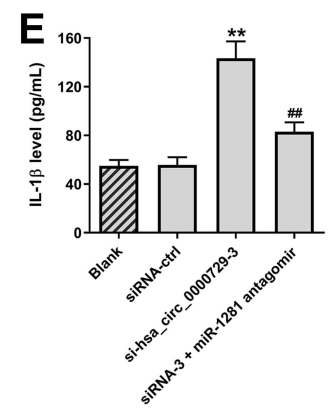

Figure 4 Hsa_circ_0000729 knockdown-induced pyroptosis in NSCLC cells was partially reversed by miR-I28I antagomir. NSCLC cells were treated with NC, si-hsa _circ_0000729-3 or si-hsa_circ_0000729-3 + miR-I28I antagomir. Then, (A) the protein levels of FOXO3, cleaved caspase I and GSDME-N in NSCLC cells were investigated by Western blot. (B-D) The relative expressions were quantified by normalizing to $\beta$-actin. (E and F) The levels of IL-I $\beta$ and IL-I 8 in supernatants of NSCLC cells were tested by ELISA. (G and $\mathbf{H}$ ) The percentage of NSCLC cell death was tested by flow cytometry. (I) The viability of NSCLC cells was detected by CCK-8 assay. $* * \mathrm{P}<0.01$ compared with control. ${ }^{\#} \mathrm{P}<0.01$ compared with si-hsa_circ_0000729-3. 
A

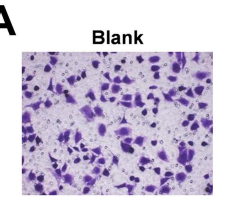

si-hsa_circ_0000729-3
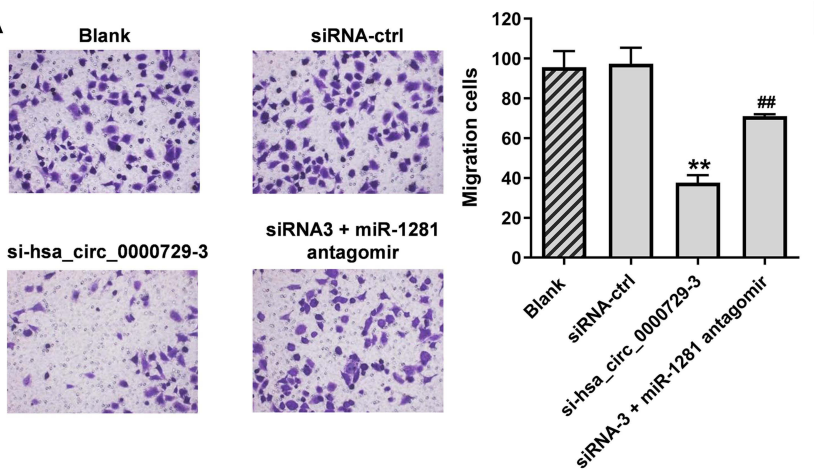

B
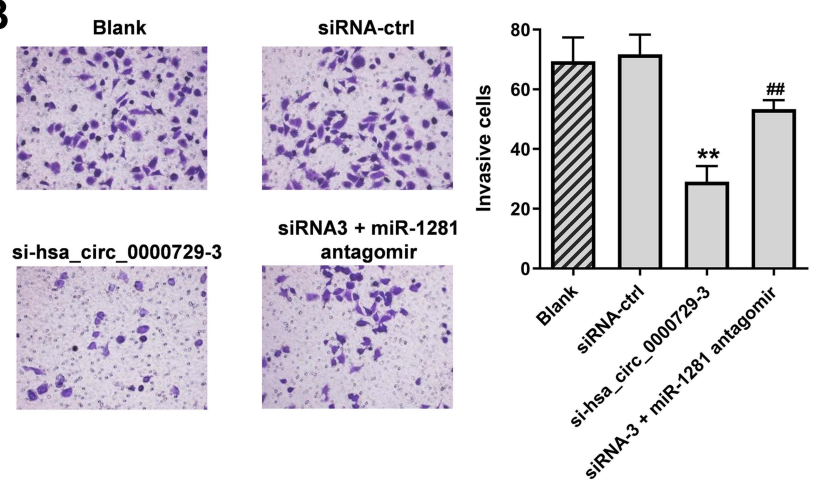
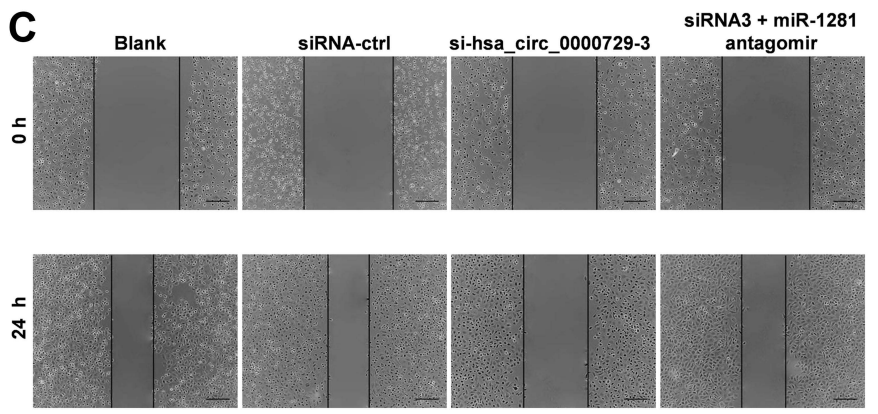

Figure 5 Silencing of hsa_circ_0000729 inhibited the migration and invasion of NSCLC cells cells were detected by transwell assay. (C) The migration of NSCLC cells was tested by wou si-hsa_circ_0000729-3.

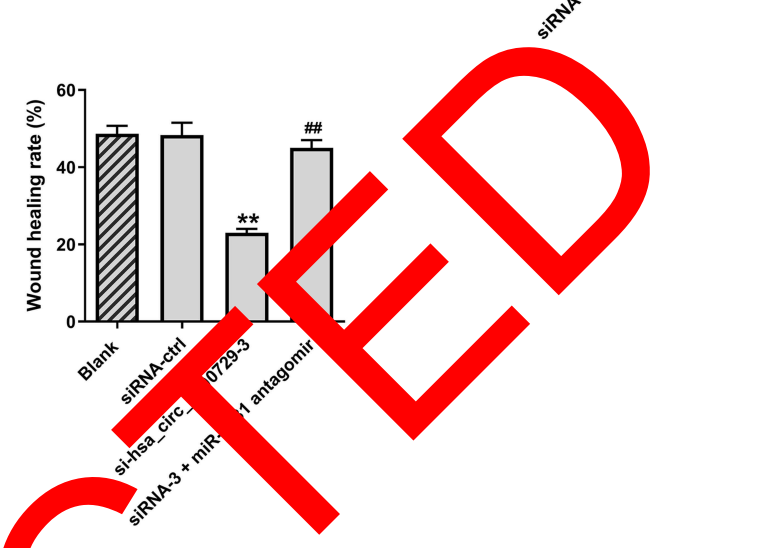

rough sponging n

- 128I. (A and B) The migration and invasion of NSCLC 0.01 compared with control. ${ }^{\#} \mathrm{P}<0.01$ compared with

in NCI-H1650 cells was significantly pcDNA3.1-hsa_circ_0000729. In ad cion, sa_circ_0000729 overexpression significently crea oum viability, migration and invasion $\mathrm{NCI}-\mathrm{H}, 50$ cells (Supplementary Figure 2B-D) $\mathrm{S}$ mary, ove pression of hsa_circ_0000729 ivcreased tho rability, migration and invasion of NCI 1650 cells.

Hsa_circ_000072, 'nockd' wn Inhibited the Tumo Gro vth o sCLC in vivo

To further vestiga finction of hsa_circ_0000729 in NSCLC in vin -nograft mice model was established. As revealed in Fig $6 \mathrm{~A}$ and $\mathrm{B}$, knockdown of hsa circ_0000729 signitreantly decreased the tumor sizes in mice. Consistently, the tumor weight of mice was obviously decreased in the presence of si-hsa _circ_0000729-3 (Figure 6C). Meanwhile, hsa_circ_0000729 silencing markedly inhibited the expression of FOXO3 and upregulated cleaved caspase 1 and GSDME-N in tumor tissues of mice (Figure 6D-G). To sum up, hsa_circ_0000729 downregulation inhibited the tumor growth of NSCLC in vivo.

\section{Discussion}

has been reported that circRNAs play vital roles in the progression of NSCLC. ${ }^{18,19}$ In this research, hsa_circ_0000729 was found to be notably upregulated in NSCLC cells, and hsa_circ_0000729 knockdown could inhibit the progression of NSCLC. A previous research indicated that hsa_circ_0000729 could act as a potential prognostic biomarker in LUAD. ${ }^{8}$ Our data was consistent to this previous research. In addition, the current study firstly explored the function of hsa_circ_0000729 in NSCLC, suggesting that hsa_circ_0000729 could act as a promoter in NSCLC.

It is reported that circRNAs could regulate the tumorigenesis of NSCLC through sponging miRNAs. For instance, Duan et al found that overexpression of hsa_circ_0074027 could lead to the development of NSCLC via mediation of miR-2467-3p; ${ }^{20} \mathrm{Lu}$ et al demonstrated that upregulation of hsa_circ_0020123 could facilitate the progression of NSCLC through binding with miR-142-3p. ${ }^{21}$ In this study, miR-1281 could be bound with hsa_circ_0000729, firstly indicating the correlation between hsa_circ_0000729 and miR-1281 in NSCLC. In addition, miR-1281 has been reported to be the 

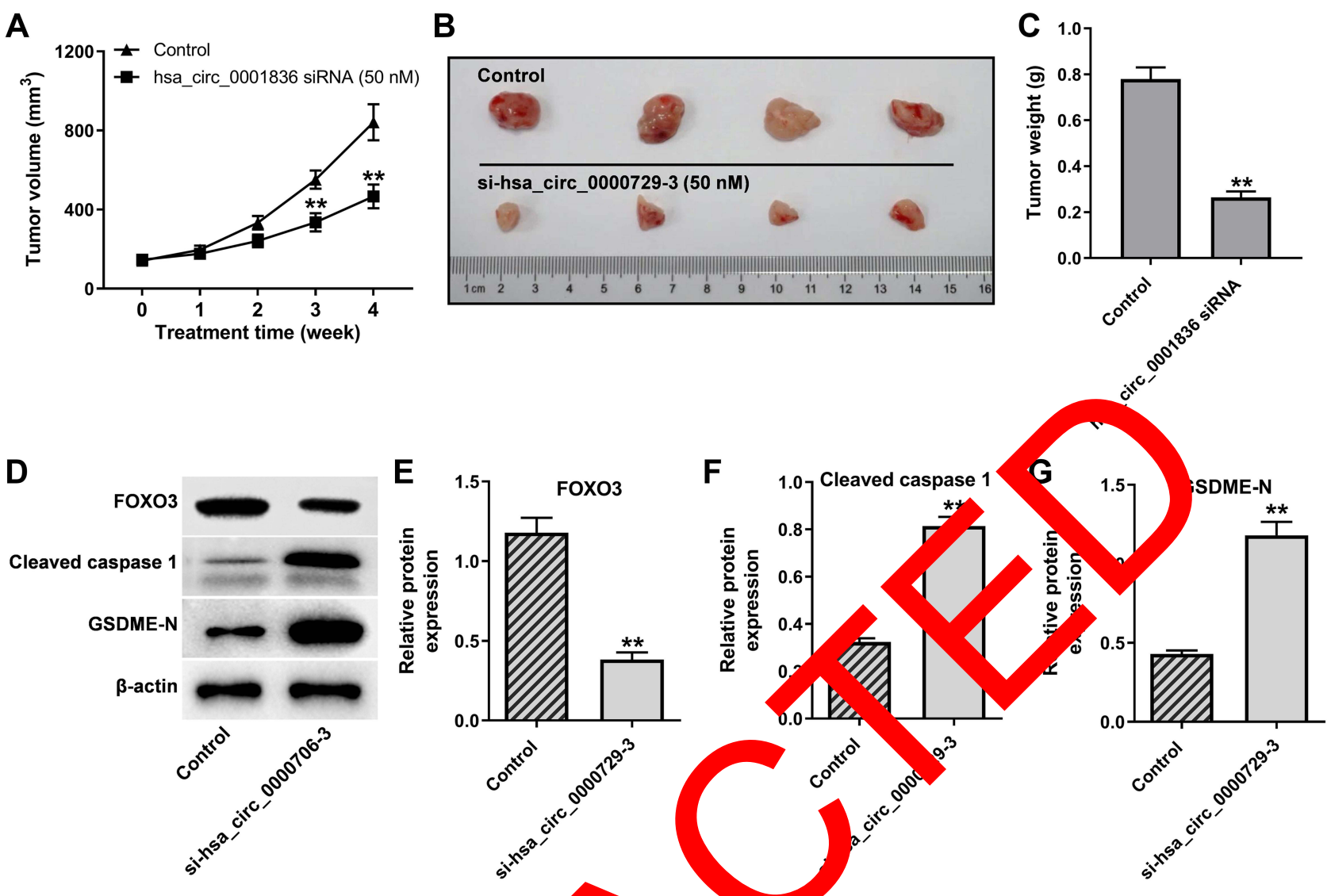

Figure 6 Hsa_circ_0000729 knockdown inhibited the tumor growth of $N$ LC in the tumor volume of mice was tested weekly. (B) The tumor tissues of mice were pictured. (C) The tumor weight of mice was determined. (D) The pr in exp investigated by Western blot. (E-G) The relative expressions wore quantifie FOXO3, cleaved caspase I and GSDME- $\mathrm{N}$ in tumor tissues of mice were malizing to $\beta$-actin. **P $<0.01$ compared with control.

inhibitor in tumors. ${ }^{22,23}$ Consistently 1281 antagomir could reverse th circ_0000729 knockdown on gested the function of miR-281 in SCLC. Thus, it could be concluded that ho circ_0000729 ight regulate the tumorigenesis of $\mathrm{N}^{\prime} \mathrm{LC}$ by ediation of miR-1281.

FOXO3 is know $b$ a key reg lator in cell growth. ${ }^{24}$ In our researcb $\mathrm{P} 3 \mathrm{~m}$ identif $\mathrm{d}$ to be the direct target of miR-128. It has b en repor that FOXO3 participate in cell pyroptos 25 ould be suggested that hsa circ_00007 knockdown could induce pyroptosis in NSCLC cells indirectly targeting FOXO3. Meanwhile, $\mathrm{Hu}$ et al found that miR-1281 inhibitor could reverse LNC01857 silencing-induced inhibition of glioma cell proliferation through upregulation of TRIM65. ${ }^{26}$ Our data were similar to this previous study. TRIM65 was known to be a promoter in the progression of cancer. Consistently, FOXO3 is known to promote the cell growth. ${ }^{27,28}$ Thereby, the similar function between FOXO3 and TRIM65 in cancer progression might result in the similarity between our study and $\mathrm{Hu}$ et al.

Caspase 1 and GSDME-N are known to be the important modulator in cell pyroptosis. ${ }^{29,30}$ Activation of caspase 1 could lead to the upregulation of GSDME-N. ${ }^{31,32}$ Meanwhile, IL-1 $\beta$ and IL-18 are also pyroptosis-related cytokines. ${ }^{33,34}$ Consistently, our study revealed that knockdown of hsa_circ_0000729 could induce pyroptosis in NSCLC cells via upregulating cleaved caspase 1, GSDME-N, IL-1 $\beta$ and IL-18.

Indeed, there are some limitations in this research as follows: 1) more miRNAs sponged by hsa_circ_0000729 remain unclear; 2) more mRNAs targeted by miR-1281 in NSCLC cells are needed to be further explored. Thereby, more investigations remain to be performed in future.

In summary, knockdown of hsa_circ_0000729 inhibits the tumorigenesis of NSCLC through mediation of miR1281/FOXO3 axis. Thus, hsa_circ_0000729 might be served as a crucial mediator in NSCLC. 


\section{Acknowledgements}

This study was supported by the National Natural Science Foundation of China(Nos. 82173382).

\section{Disclosure}

These authors declare no competing interests in this research.

\section{References}

1. Xing JJ, Hou JG, Ma ZN, et al. Ginsenoside $\mathrm{Rb} 3$ provides protective effects against cisplatin-induced nephrotoxicity via regulation of AMPK-/ mTOR-mediated autophagy and inhibition of apoptosis in vitro and in vivo. Cell Prolif. 2019;52(4):e12627. doi:10.1111/cpr.12627

2. Wen Q, Jiao X, Kuang F, et al. FoxO3a inhibiting expression of EPS8 to prevent progression of NSCLC: a new negative loop of EGFR signaling. EBioMedicine. 2019;40:198-209. doi:10.1016/j.ebiom.2019.01.053

3. Zhang Y, Zhao H, Zhang L. Identification of the tumorsuppressive function of circular RNA FOXO3 in nonsmall cell lung cancer through sponging miR155. Mol Med Rep. 2018;17(6):7692-7700.

4. Chen G, Yu L, Dong H, Liu Z, Sun Y. MiR-182 enhances radioresistance in non-small cell lung cancer cells by regulating FOXO3. Clin Exp Pharmacol Physiol. 2019;46(2):137-143. doi:10.1111/ 1440-1681.13041

5. Zhu Y, Zhao P, Sun L, et al. Overexpression of circRNA SNRK targets miR-103-3p to reduce apoptosis and promote cardiac repair through GSK3beta/beta-catenin pathway in rats with myocardial infarction. Cell Death Discov. 2021;7(1):84. doi:10.1038/s4142 021-00467-3

6. Yi S, Li Z, Wang X, Du T, Chu X. Circ_0001806 promotes th proliferation, migration and invasion of NSCLC cells through miR-1182/NOVA2 axis. Cancer Manag Res. 2021. doi:10.2147/CMAR.S290059

7. Wang Y, Wang Z, Lu J, Zhang H. Circular RN Hum Cell. 2021;34(4):1174-84

8. Li S, Sun X, Miao S, et al. hsa_circ 0 29, a potentia rognostic biomarker in lung adenocarcin a. Aac Cancer. 2018;9 (8):924-930. doi:10.1111/1759-7714.12761

9. Zhu J, Ao H, Liu M, Cao K a J. UBE2T pron s autophagy via the $\mathrm{p} 53 / \mathrm{AMPK} / \mathrm{mTOR}$ si ding pathway in lung menocarcinoma. $J$ Transl Med. 2021;19 374. do .1186/s12967-021-03056-1

10. Tan Y, Sun R, Liu L, et a Tu suppres ${ }^{\circ}$ DRD2 facilitates M1 macrophages and icts $\mathrm{N}$ appaB sig ang to trigger pyroptosis in breast cand Thero stics. $\quad$; 1 1):5214-5231. doi:10.7150/

thno. 5832

11. Uematsu vuchiy glycolytic ano ave alterations promote pyroptosis and prevent the spread of Listerka onocytogenes infection in macrophages. Cell Death Dis. 2021;12(4):404. i:10.1038/s41419-021-03691-y

12. Faria SS, Costantìn S, de Lima VCC, et al. NLRP3 inflammasome-mediated cytokine production and pyroptosis cell death in breast cancer. J Biomed Sci. 2021;28(1):26. doi:10.1186/ s12929-021-00724-8

13. Sok SPM, Ori D, Wada A, et al. 1'-acetoxychavicol acetate inhibits NLRP3-dependent inflammasome activation via mitochondrial ROS suppression. Int Immunol. 2021;33(7):373-386. doi:10.1093/intimm/ dxab016

14. Peng Z, Wang P, Song W, et al. GSDME enhances Cisplatin sensitivity to regress non-small cell lung carcinoma by mediating pyroptosis to trigger antitumor immunocyte infiltration. Signal Transduct Target Ther. 2020;5(1):159. doi:10.1038/s41392-020-00274-9
15. Chen S, Zhou C, Yu H, et al. 27-Hydroxycholesterol contributes to lysosomal membrane permeabilization-mediated pyroptosis in co-cultured SH-SY5Y cells and C6 cells. Front Mol Neurosci. 2019;12:14. doi:10.3389/fnmol.2019.00014

16. Liu H, Zhang Y, Song W, Sun Y, Jiang Y. Osteopontin N-terminal function in an abdominal aortic aneurysm from apolipoprotein E-deficient mice. Front Cell Dev Biol. 2021;9:681790. doi:10.3389/ fcell.2021.681790

17. Sun W, Liu S, Huang X, Yuan R, Yu J. Cytokine storms and pyroptosis are primarily responsible for the rapid death of mice infected with pseudorabies virus. $R$ Soc Open Sci. 2021;8(8):210296. doi: $10.1098 /$ rsos. 210296

18. Yue Q, Xu Y, Deng X, et al. Circ-PITX1 promotes the progression of non-small cell lung cancer through regulating the miR-1248/CCND2 axis. Onco Targets Ther. 2021;14:1807 doi:10.2147/OTT. S286820

19. Zhao Q, Zhao F, Liu C, Xu T, S K. LncRNA OXD2-AS1 promotes cell proliferation and invas of fibroblast- te synoviocytes by regulation of miR-3 Sp/PIA vathway rheumatoid arthritis. Autoimmunity 2021;54(5):2 263 doi:10.1080/ 08916934.2021.1919870

20. Duan Z, Wei S, Liu Y. 00727 contributes to non-small cell lung cancer prog oron the positive modulating RHOA via sequestering -2467-3p. Bio rg Biomembr. 2021;53 (2):223-233 of: $007 / \mathrm{s} 10863-0 \quad 0876-6$

21. Lu J, Ma X, Lin J, Ho Circ_0020123 increases ZFX expression to facilitan-small co lung cancer progression by sponging m. 142-3p. Cancer Mana Res. 2021;13:1687-1698. doi:10.2147/ AAR.S295595

22. n LY, Shi $\mathrm{k}$ Xu D, et al. LncRNA GIHCG regulates n oRNA-1281 nd promotes malignant progression of breast can Eur Re led Pharmacol Sci. 2019;23(24):10842-10850.

Liu G, M. L, Qiao M, Wang F. Lnc-GIHCG promotes cell prolifand migration in gastric cancer through miR- 1281 adsorpwon. Mol Genet Genomic Med. 2019;7(6):e711. doi:10.1002/ $\operatorname{mgg} 3.711$

4. Bi C, Wang G. LINC00472 suppressed by ZEB1 regulates the miR-23a-3p/FOXO3/BID axis to inhibit the progression of pancreatic cancer. J Cell Mol Med. 2021;25(17):8312-8328. doi:10.1111/ jcmm. 16784

25. Liang $\mathrm{C}$, Liu $\mathrm{Y}, \mathrm{Xu} \mathrm{H}$, et al. Exosomes of human umbilical cord MSCs protect against hypoxia/reoxygenation-induced pyroptosis of cardiomyocytes via the miRNA-100-5p/FOXO3/NLRP3 pathway. Front Bioeng Biotechnol. 2020;8:615850. doi:10.3389/ fbioe. 2020.615850

26. Hu G, Liu N, Wang H, Wang Y, Guo Z. LncRNA LINC01857 promotes growth, migration, and invasion of glioma by modulating miR-1281/TRIM65 axis. $J$ Cell Physiol. 2019;234(12):22009-22016. doi: $10.1002 /$ jcp. 28763

27. Wu X, Chang SC, Jin J, Gu W, Li S. NLRP3 in fl ammasome mediates chronic intermittent hypoxia-induced renal injury implication of the microRNA-155/FOXO3a signaling pathway. $J$ Cell Physiol. 2018;233(12):9404-9415. doi:10.1002/jcp.26784

28. Yin Y, Wang J, Zhao X, et al. Overexpressed FOXO3 improves inflammatory status in mice by affecting NLRP3-mediated cell coronation in necrotizing colitis mice. Biomed Pharmacother. 2020;125:109867. doi:10.1016/j.biopha.2020.109867

29. Kim N, Choi JG, Ju IG. et al. A novel nutritional mixture, MBN, prevents memory impairment via inhibiting NLRP3 inflammasome formation in 5xFAD transgenic mice. Nutr Neurosci. 2021;1-8. doi:10.1080/1028415X.2021.1913952

30. Lou Y, Miao J, Li F, Ding J, Wang L. Maternal smoking during pregnancy aggravated muscle phenotype in FHL1(-/y) offspring mice similar to congenital clubfoot through P2RX7-mediated pyroptosis. Toxicol Lett. 2021;345:54-60. doi:10.1016/j. toxlet.2021.04.014 
31. Xia W, Li Y, Wu M, et al. Gasdermin E deficiency attenuates acute kidney injury by inhibiting pyroptosis and inflammation. Cell Death Dis. 2021;12(2):139. doi:10.1038/s41419-021-03431-2

32. Li Y, Yuan Y, Huang ZX, et al. GSDME-mediated pyroptosis promotes inflammation and fibrosis in obstructive nephropathy. Cell Death Differ. 2021;28:2333-2350.

33. Garcia-Bonilla L, Sciortino R, Shahanoor Z, et al. Role of microglial and endothelial CD36 in post-ischemic inflammasome activation and interleukin-1beta-induced endothelial activation. Brain Behav Immun. 2021;95:489-501. doi:10.1016/j.bbi.2021.04.010
34. Zeng Y, Yan Wang C, Xu J, Le Xu X. Overexpression of retinoid $\mathrm{X}$ receptor beta provides protection against oxidized low-density lipoprotein-induced inflammation via regulating PGClalpha-dependent mitochondrial homeostasis in endothelial cells. Biochem Pharmacol. 2021;188:114559. doi:10.1016/j.bcp.2021.114559

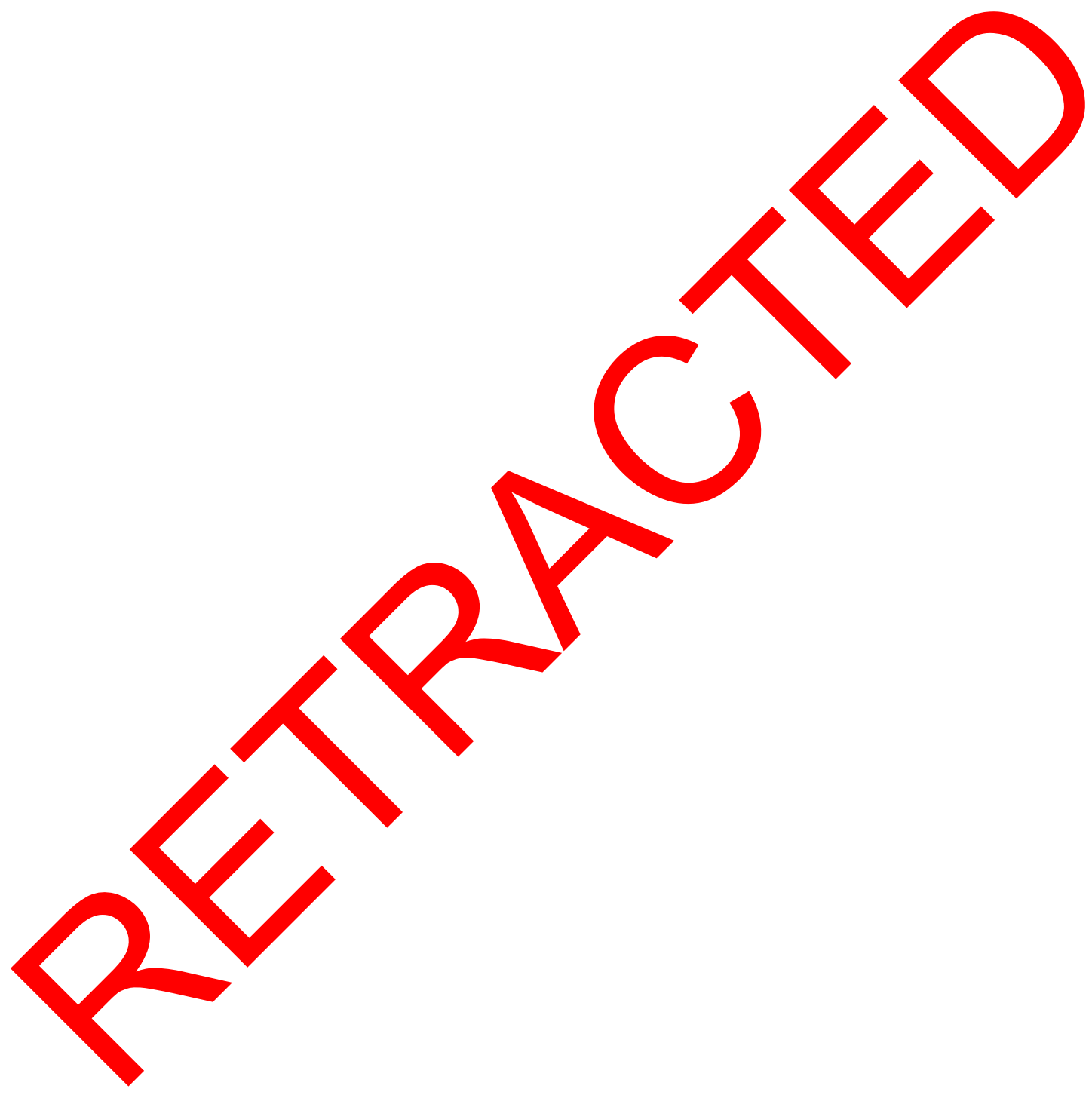

Cancer Management and Research

Dovepress

\section{Publish your work in this journal}

Cancer Management and Research is an international, peer-reviewed open access journal focusing on cancer research and the optimal use of preventative and integrated treatment interventions to achieve improved outcomes, enhanced survival and quality of life for the cancer patient.
The manuscript management system is completely online and includes a very quick and fair peer-review system, which is all easy to use. Visit http://www.dovepress.com/testimonials.php to read real quotes from published authors. 